\title{
APPROXIMATION BY SEVERAL RATIONALS
}

\author{
IGOR E. SHPARLINSKI
}

(Received 15 August 2007)

\begin{abstract}
Following T. H. Chan, we consider the problem of approximation of a given rational fraction $a / q$ by sums of several rational fractions $a_{1} / q_{1}, \ldots, a_{n} / q_{n}$ with smaller denominators. We show that in the special cases of $n=3$ and $n=4$ and certain admissible ranges for the denominators $q_{1}, \ldots, q_{n}$, one can improve a result of T. H. Chan by using a different approach.
\end{abstract}

2000 Mathematics subject classification: 11J04, 11N25.

Keywords and phrases: rational approximations, multivariate congruences.

\section{Introduction}

Chan [1] has recently considered the question of approximating real numbers by sums of several rational fractions $a_{1} / q_{1}, \ldots, a_{n} / q_{n}$ with bounded denominators.

In the special case of $n=3$ the result of Chan [1] can be reformulated as follows. Given two integers $a$ and $q \geqslant 1$, for any $Q \geqslant q$ there are integers $a_{i}$ and $q_{i}$ with $1 \leqslant q_{i} \leqslant Q^{1 / 2+o(1)}, i=1,2,3$, and such that

$$
\left|\frac{a}{q}-\frac{a_{1}}{q_{1}}-\frac{a_{2}}{q_{2}}-\frac{a_{3}}{q_{3}}\right| \leqslant \frac{1}{q Q^{1+o(1)}} .
$$

We remark that the numerators $a_{1}, a_{2}, a_{3}$ can be negative.

In this paper we use a different approach to show that when $Q$ is large enough, that is, when $Q \geqslant q^{2+\varepsilon}$, the same result holds with $1 / 3$ instead of $1 / 2$. We also obtain more explicit constants.

Similarly, for $n=4$, we see from [1] that for any $Q \geqslant q$ there are integers $a_{i}$ and $q_{i}$ with $1 \leqslant q_{i} \leqslant Q^{2 / 5+o(1)}, i=1,2,3,4$, and such that

$$
\left|\frac{a}{q}-\frac{a_{1}}{q_{1}}-\frac{a_{2}}{q_{2}}-\frac{a_{3}}{q_{3}}-\frac{a_{4}}{q_{4}}\right| \leqslant \frac{1}{q Q^{1+o(1)}} .
$$

In this case, under the same condition $Q \geqslant q^{2+\varepsilon}$ we replace $2 / 5$ with $1 / 4$.

This work was supported in part by ARC grant DP0556431.

(C) 2008 Australian Mathematical Society 0004-9727/08 \$A2.00+0.00 
Our approach is based on a result of [3] about the uniformity of distribution in residue classes of rather general products. More precisely, it is shown in [3] that for any set $\mathcal{X} \in[1, X]$ of integers $x$ with $\operatorname{gcd}(x, q)=1$ and for any interval $[Z+1, Z+Y]$, for the number $M_{u, q}(\mathcal{X} ; Y, Z)$ of solutions to the congruence

$$
u \equiv x y \quad(\bmod q), \quad x \in \mathcal{X}, y \in[Z+1, Z+Y]
$$

we have

$$
\sum_{u=1}^{q}\left|M_{u, q}(\mathcal{X} ; Y, Z)-\# \mathcal{X} \frac{Y}{q}\right|^{2} \leqslant \# \mathcal{X}(X+Y) q^{o(1)}
$$

\section{Approximation by three rationals}

THEOREM 1. Let $a$ and $q \geqslant 1$ be integers with $\operatorname{gcd}(a, q)=1$. For any fixed $\varepsilon>0$ and sufficiently large $q$, for any integer $Q \geqslant q^{2+\varepsilon}$ there are integers $a_{i}$ and $q_{i}$ with $1 \leqslant q_{i} \leqslant 2 Q^{1 / 3}, i=1,2,3$, and such that

$$
\left|\frac{a}{q}-\frac{a_{1}}{q_{1}}-\frac{a_{2}}{q_{2}}-\frac{a_{3}}{q_{3}}\right| \leqslant \frac{1}{q Q}
$$

holds.

PROOF. We note that it is enough to show that there are positive integers $q_{1}, q_{2}, q_{3}$ $\leqslant 2 Q^{1 / 3}$ with

$$
q_{1} q_{2} q_{3} \geqslant Q
$$

such that

$$
\operatorname{gcd}\left(q_{1}, q_{2}\right)=\operatorname{gcd}\left(q_{1}, q_{3}\right)=\operatorname{gcd}\left(q_{2}, q_{3}\right)=1
$$

and

$$
a q_{1} q_{2} q_{3} \equiv 1 \quad(\bmod q) .
$$

Indeed, from (4) we conclude that $a q_{1} q_{2} q_{3}=1+b q$ for some integer $b$. Since (3) implies that

$$
\operatorname{gcd}\left(q_{1} q_{2}, q_{1} q_{3}, q_{2} q_{3}\right)=1
$$

then

$$
b=a_{1} q_{2} q_{3}+a_{2} q_{1} q_{3}+a_{3} q_{1} q_{2},
$$

for some integers $a_{1}, a_{2}, a_{3}$. Thus

$$
\left|\frac{a}{q}-\frac{a_{1}}{q_{1}}-\frac{a_{2}}{q_{2}}-\frac{a_{3}}{q_{3}}\right|=\frac{1}{q q_{1} q_{2} q_{3}} \leqslant \frac{1}{q Q} .
$$

Let us put $R=\left\lfloor 2 Q^{1 / 3}\right\rfloor$. We may assume that $R<q$ since otherwise we simply choose $a_{1}=1, a_{2}=a_{3}=0, q_{1}=q, q_{2}=q_{3}=1$. 
We now consider:

- $\quad$ the set $\mathcal{S}$ consisting of integers $s \in[R / 3, R / 2)$;

- $\quad$ the set $\mathcal{P}$ consisting of primes $p \in[R / 2,3 R / 4)$ with $\operatorname{gcd}(p, q)=1$;

- $\quad$ the set $\mathcal{L}$ consisting of primes $\ell \in[3 R / 4, R]$ with $\operatorname{gcd}(\ell, q)=1$.

Since $q$ may have at most $O(\log q)$ prime divisors, by the prime number theorem we see that

$$
\# \mathcal{S}, \# \mathcal{P}, \# \mathcal{L} \geqslant R^{1+o(1)}
$$

Clearly, if we take $q_{1}=s \in \mathcal{S}, q_{2}=p \in \mathcal{P}$ and $q_{3}=\ell \in \mathcal{L}$ then (3) is satisfied and we also have (2). Thus it is enough to show that the congruence

$$
s p \ell \equiv 1 \quad(\bmod q), \quad s \in \mathcal{S}, p \in \mathcal{P}, \ell \in \mathcal{L},
$$

has a solution. For an integer $u \in[1, q]$ we denote by $N(u)$ the number of solutions to the congruence

$$
s p \equiv u \quad(\bmod q), \quad s \in \mathcal{S}, p \in \mathcal{P} .
$$

Let $\mathcal{U}$ be the set of integers $u \in[1, q]$ for which the above congruence has a solution, that is, $N(u)>0$. It is enough to show that the congruence

$$
u \ell \equiv 1 \quad(\bmod q), \quad u \in \mathcal{U}, \ell \in \mathcal{L}
$$

has a solution.

Also let $\mathcal{V}$ be the set of remaining integers $u \in[1, q]$ with $N(u)=0$. It follows from [3] that

$$
\sum_{u=1}^{q}\left|N(u)-\frac{\# \mathcal{S} \# \mathcal{P}}{q}\right|^{2} \leqslant R^{2} q^{o(1)}
$$

see (1). Hence

$$
\# \mathcal{V}\left(\frac{\# \mathcal{S} \# \mathcal{P}}{q}\right)^{2} \leqslant R^{2} q^{o(1)},
$$

which implies that $\# \mathcal{V} \leqslant R^{-2} q^{2+o(1)}$. Recalling that $R \geqslant 2 Q^{1 / 3}-1 \geqslant q^{2 / 3+\varepsilon / 3}$, we see that

$$
\# \mathcal{L}-\# \mathcal{V}=R^{1+o(1)}-R^{-2} q^{2+o(1)}>0
$$

provided that $q$ is large enough. Therefore the congruence (6) has a solution, which concludes the proof. 


\section{Approximation by four rationals}

We now use a similar approach for approximations by four rational fractions.

THEOREM 2. Let $a$ and $q \geqslant 1$ be integers with $\operatorname{gcd}(a, q)=1$. For any fixed $\varepsilon>0$ and sufficiently large $q$, for any integer $Q \geqslant q^{2+\varepsilon}$ there are integers $a_{i}$ and $q_{i}$ with $1 \leqslant q_{i} \leqslant 2 Q^{1 / 4}, i=1,2,3$, and such that

$$
\left|\frac{a}{q}-\frac{a_{1}}{q_{1}}-\frac{a_{2}}{q_{2}}-\frac{a_{3}}{q_{3}}-\frac{a_{4}}{q_{4}}\right| \leqslant \frac{1}{q Q}
$$

holds.

PROOF. We proceed as in the proof of Theorem 1. In particular, we see that it is enough to show that there are positive integers $q_{1}, q_{2}, q_{3}, q_{4} \leqslant 2 Q^{1 / 4}$ with

$$
q_{1} q_{2} q_{3} q_{4} \geqslant Q
$$

such that

$$
\operatorname{gcd}\left(q_{i}, q_{j}\right)=1, \quad 1 \leqslant i<j \leqslant 4
$$

and

$$
a q_{1} q_{2} q_{3} q_{4} \equiv 1 \quad(\bmod q) .
$$

Let us put $R=\left\lfloor 2 Q^{1 / 4}\right\rfloor$. As before, we remark that we may assume that $R<q$ since otherwise the result is trivial.

We now consider:

- $\quad$ the set $\mathcal{S}$ consisting of integers $s \in[R / 4, R / 3)$;

- $\quad$ the set $\mathcal{P}$ consisting of primes $p \in[R / 3,2 R / 3)$ with $\operatorname{gcd}(p, q)=1$;

- $\quad$ the set $\mathcal{L}$ consisting of primes $\ell \in[2 R / 3,3 R / 4)$ with $\operatorname{gcd}(\ell, q)=1$;

- $\quad$ the set $\mathcal{R}$ consisting of primes $r \in[3 R / 4, R]$ with $\operatorname{gcd}(r, q)=1$.

Again, by the prime number theorem,

$$
\# \mathcal{S}, \# \mathcal{P}, \# \mathcal{L}, \# \mathcal{R} \geqslant R^{1+o(1)} .
$$

Clearly, if we take $q_{1}=s \in \mathcal{S}, q_{2}=p \in \mathcal{P}, q_{3}=\ell \in \mathcal{L}$ and $q_{4}=r \in \mathcal{R}$ then (8) is satisfied and we also have (7). Thus it is enough to show that the congruence

$$
s p \ell r \equiv 1 \quad(\bmod q), \quad s \in \mathcal{S}, p \in \mathcal{P}, \ell \in \mathcal{L}, r \in \mathcal{R},
$$

has a solution.

As in the proof of Theorem 1 we note the set $\mathcal{V}$ of integers $u \in[1, q]$ for which the congruence (5) does not have a solution is of cardinality $\# \mathcal{V} \leqslant R^{-2} q^{2+o(1)}$.

Let $\mathcal{W}$ be the set of integers $w \in[1, q]$ which are of the form $w \equiv \ell r(\bmod q)$ with $\ell \in \mathcal{L}$ and $r \in \mathcal{R}$. We note that $\# \mathcal{L} \# \mathcal{R}=R^{2+o(1)}$ products $\ell r$ are distinct integers in 
the interval $\left[1, R^{2}\right]$. Since there are at most $R^{2} / q+1$ integers $t \in\left[1, R^{2}\right]$ in the same residue class modulo $q$, we obtain

$$
\# \mathcal{W} \geqslant R^{2+o(1)}\left(R^{2} / q+1\right)^{-1} .
$$

Since $R^{2} \geqslant\left(2 Q^{1 / 4}-1\right)^{2} \geqslant Q^{1 / 2} \geqslant q^{1+\varepsilon / 2}$ (provided $q$ is large enough) we see that $R^{2} / q+1 \leqslant 2 R^{2} / 1$. Hence $\# \mathcal{W}=q^{1+o(1)}$. We now see that

$$
\# \mathcal{W}-\# \mathcal{V}=q^{1+o(1)}-R^{-2} q^{2+o(1)}>0
$$

provided that $q$ is large enough. The desired result now follows.

We remark that in both Theorems 1 and 2 the coefficient 2 in the bound on the denominators can be replaced by any constant $c>1$.

\section{Comments}

It is natural to try to use (1) to improve the corresponding bound from [1] for larger values of $n$ too. Although some results can be obtained in this way, for $n \geqslant 5$ we have not been able to achieve this. In fact, it seems quite plausible that for $n \geqslant 5$, instead of using the bound (1) from [3], one can study the solvability of the congruence

$$
q_{1} \cdots q_{n} \equiv 1 \quad(\bmod q)
$$

with 'small' $q_{1}, \ldots, q_{n}$ by using bounds of multiplicative character sums in the same style as in $[2,4]$.

\section{Acknowledgement}

The author is grateful to Tsz Ho Chan for useful discussions.

\section{References}

[1] T. H. Chan, 'Approximating reals by sums of rationals', Preprint, 2007 (available from http://arxiv.org/abs/0704.2805).

[2] I. E. Shparlinski, 'On the distribution of points on multidimensional modular hyperbolas', Proc. Japan Acad. Sci., Ser. A 83 (2007), 5-9.

[3] I. E. Shparlinski, 'Distribution of inverses and multiples of small integers and the Sato-Tate conjecture on average', Michigan Math. J. to appear.

[4] I. E. Shparlinski, 'On a generalisation of a Lehmer problem', Preprint, 2006 (available from http://arxiv.org/abs/math/0607414).

IGOR E. SHPARLINSKI, Department of Computing, Macquarie University, Sydney, NSW 2109, Australia

e-mail: igor@ics.mq.edu.au 